\title{
Closure of a bronchopleural fistula after extended right pneumonectomy after induction chemotherapy with BioGlue surgical adhesive
}

\author{
Loïc Lang-Lazdunski, MD, PhD, FRCS, London, United Kingdom
}

B ronchopleural fistula (BPF) after pneumonectomy remains a major challenge for thoracic surgeons. ${ }^{1,2}$ Conventional treatment combines chest tube drainage with intravenous antibiotic therapy followed by one or several of the following procedures: thoracotomy for debridement of the pleural cavity and manual closure of the bronchial stump, sternotomy and transpericardial closure of the main bronchial stump, intrathoracic transposition of a pedicled skeletal muscle or omental flap, video-assisted closure through a short cervicotomy, and open window thoracostomy. ${ }^{1-3}$

My colleagues and I intended to seal a BPF after right pneumonectomy using BioGlue surgical adhesive (CryoLife Inc, Kennesaw, Ga) in a patient who had received three cycles of induction chemotherapy and wished not to have any major procedure performed.

\section{Clinical Summary}

A 53-year-old woman was referred to our department with a potentially operable bronchial adenocarcinoma. Bronchoscopy, chest computed tomography, and positron emission tomography had revealed a T4 N2 M0 tumor in the right upper lobe and right main bronchus invading the superior vena cava and azygos arch (Figure 1). The patient received three cycles of induction chemotherapy with gemcitabine and cisplatin, and repeat chest computed tomography demonstrated a good response, allowing us to undertake a right pneumonectomy.

Extended right pneumonectomy was performed, including en bloc resection of the right main bronchus and azygos arch. Radical mediastinal lymphadenectomy was performed. The right main bronchus was cut $5 \mathrm{~mm}$ from the carina. The bronchial stump was closed with interrupted 3-0 polydioxanone suture (PDS; Ethicon, Inc, Somerville, NJ) and was reinforced by a pedicled intercostal muscle flap. The postoperative course was uneventful and the patient was discharged home after 9 days. Histopathologic examination revealed a pT2 N0 M0 bronchial adenocarcinoma. All margins were clear.

\footnotetext{
From the Department of Thoracic Surgery, Guy's Hospital and King's College, London, United Kingdom.

Received for publication June 12, 2006; accepted for publication Aug 8, 2006.

Address for reprints: Loïc Lang-Lazdunski, MD, PhD, FRCS, Department of Thoracic Surgery, Guy's Hospital, St Thomas St, London SE1 9RT, United Kingdom (E-mail: loic.lang-lazdunski@gstt.nhs.uk).

J Thorac Cardiovasc Surg 2006;132:1497-8

$0022-5223 / \$ 32.00$

Copyright $\odot 2006$ by The American Association for Thoracic Surgery doi:10.1016/j.jtcvs.2006.08.029
}

The patient was readmitted 2 weeks later with empyema. A chest drain was inserted and the patient was started on a regimen of intravenous cefotaxime and metronidazole. Pleural fluid grew Streptococcus milleri. Examination with a flexible bron-

choscope revealed no obvious fistula on the right main bronchial stump. The patient rejected any major procedure but agreed to reoperation for debridement of the cavity and revision of bronchial stump.

The thoracotomy was partly reopened, and the pleural cavity was debrided with video-assistance and copiously washed out with povidone-iodine and hydrogen peroxide. The bronchial stump was carefully dissected, and positive-pressure ventilation revealed a small BPF. Five milliliters of the albumin-glutaraldehyde tissue adhesive BioGlue was injected onto the bronchial stump, and the minithoracotomy was closed in layers. A chest drain was inserted and remained on water seal for 7 days. The patient was discharged home receiving cephadroxil and metronidazole with the drain connected to a Portex bag (Portex, Inc, Keene, NH). The chest drain was removed 2 weeks later and antibiotics were discontinued. There has been no recurrence of BPF or empyema after 2 years and the patient lives a normal life.

\section{Discussion}

The mortality associated with BPF after pneumonectomy ranges between $11 \%$ and $40 \%$ in recent series, ${ }^{1,2,4}$ justifying an aggressive approach. Current techniques include drainage and debridement of the cavity, open window thoracostomy, transpericardial closure of the bronchial stump, and intrathoracic muscle or omental flap transposition. ${ }^{1}$ Recently, Azorin and associates ${ }^{3}$ proposed a less invasive approach involving closure of the bronchial stump through a cervicotomy using a video-mediastinoscope and an endolinear stapler.

None of these techniques was an option in our patient, who had a short right main bronchial stump and who refused any major procedure. Therefore, we decided to debride the cavity and seal any eventual BPF with BioGlue surgical adhesive.

BioGlue adhesive consists of a $10 \%$ glutaraldehyde solution and a $45 \%$ bovine serum albumin solution binding to each other, to cell surface proteins, and to the extracellular matrix. ${ }^{5}$ The reaction is spontaneous and immediate and results in a strong but flexible bond reabsorbing in approximately 2 years. ${ }^{5}$ Herget and associates $^{6}$ have shown that BioGlue adhesive is progressively replaced by fibrous tissue in bronchial anastomoses and that healing is not complicated by foreign body reaction or tissue granula- 


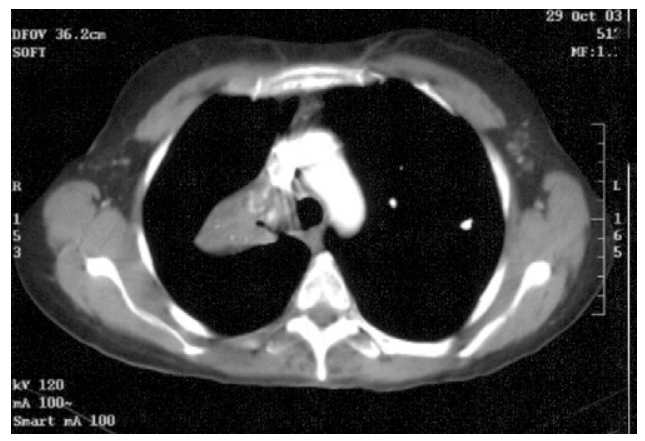

Figure 1. Chest computed tomographic scan before induction chemotherapy demonstrating a tumor involving the right upper lobe bronchus, distal right main bronchus, and superior vena cava at the level of the azygos arch. Mediastinal lymph node station 4 is involved as well.

tion after 12 weeks. BioGlue adhesive does not possess the potential histotoxicity of formaldehyde. ${ }^{7}$ Biocompatibility is better than that of cyanoacrylate, and by comparison fibrin glues have a relatively low adhesive strength. ${ }^{5}$

Recently, Potaris, Mihos, and Gakidis ${ }^{5}$ have used BioGlue adhesive to seal air leaks and BPFs 38 patients, with no occurrence of empyema. This group has reported closure of a BPF after right pneumonectomy, using BioGlue adhesive injected trough a rigid bronchoscope.
Considering these encouraging results, we suggest that BioGlue adhesive be used to seal small BPFs after pneumonectomy, in association with video-assisted debridement of the pleural cavity. Where this fails, open window thoracostomy or other techniques remain an option. Although this technique needs to be evaluated in a larger cohort of patients, this may represent a less invasive approach, particularly in debilitated patients.

\section{References}

1. Regnard JF, Alifano M, Puyo P, Fares E, Magdeleinat P, Levasseur P. Open window thoracostomy followed by intrathoracic flap transposition in the treatment of empyema complicating pulmonary resection. $J$ Thorac Cadiovasc Surg. 2000;120:270-5.

2. Gharagozloo F, Trachiotis G, Wolfe A, DuBree KJ, Cox JL. Pleural space irrigation and modified Clagett procedure for the treatment of early postpneumonectomy empyema. J Thorac Cardiovasc Surg. 1998; 116:943-8.

3. Azorin JF, Francisci MP, Tremblay B, Larmignat P, Carvaillo D. Closure of a postpneumonectomy main bronchus fistula using VideoAssisted Mediastinal Surgery. Chest. 1996;109:1097-8.

4. Deschamps C, Allen MS, Trastek VF, Pairolero PC. Empyema following pulmonary resection. Chest Surg Clin North Am. 1994;4:583-92.

5. Potaris K, Mihos P, Gakidis I. Preliminary results with the use of an albumin-glutaraldehyde tissue adhesive in lung surgery. Med Sci Monit. 2003;9:79-83.

6. Herget GW, Kassa M, Riede UN, Lu Y, Brethner L, Hasse J. Experimental use of an albumin-glutaraldehyde tissue adhesive for sealing pulmonary parenchyma and bronchial anastomoses. Eur J Cardiothorac Surg. 2001;19:4-9.

7. Hewitt CW, Marra SW, Kann BR, Tran HS, Puc MM, Chrzanowski FA $\mathrm{Jr}$, et al. BioGlue surgical adhesive for thoracic aortic repair during coagulopathy: efficacy and histopathology. Ann Thorac Surg. 2001;71: 1609-12. 